\title{
Association of peripheral blood DNA methylation level with Alzheimer's disease progression
}

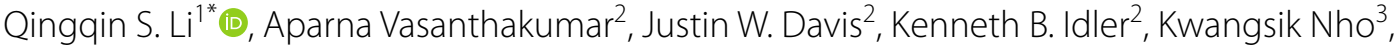 \\ Jeffrey F. Waring ${ }^{2}$ and Andrew J. Saykin ${ }^{3}$ for the Alzheimer's Disease Neuroimaging Initiative (ADNI)
}

\begin{abstract}
Background: Identifying biomarkers associated with Alzheimer's disease (AD) progression may enable patient enrichment and improve clinical trial designs. Epigenome-wide association studies have revealed correlations between DNA methylation at cytosine-phosphate-guanine $(\mathrm{CpG})$ sites and AD pathology and diagnosis. Here, we report relationships between peripheral blood DNA methylation profiles measured using Infinium ${ }^{\circledR}$ MethylationEPIC BeadChip and AD progression in participants from the Alzheimer's Disease Neuroimaging Initiative (ADNI) cohort.

Results: The rate of cognitive decline from initial DNA sampling visit to subsequent visits was estimated by the slopes of the modified Preclinical Alzheimer Cognitive Composite (mPACC; PACC $_{\text {digit }}$ and PPACC $_{\text {trailsB }}$ ) and Clinical Dementia Rating Scale Sum of Boxes (CDR-SB) plots using robust linear regression in cognitively normal (CN) participants and patients with mild cognitive impairment $(\mathrm{MCl})$, respectively. In addition, diagnosis conversion status was assessed using a dichotomized endpoint. Two $\mathrm{CpG}$ sites were significantly associated with the slope of mPACC in CN participants $\left(P<5.79 \times 10^{-8}\right.$ [Bonferroni correction threshold]); cg00386386 was associated with the slope of mPACC digit and cg09422696 annotated to RP11-661A12.5 was associated with the slope of CDR-SB. No significant CpG sites associated with diagnosis conversion status were identified. Genes involved in cognition and learning were enriched. A total of 19,13 , and 5 differentially methylated regions (DMRs) associated with the slopes of $\mathrm{MPACC}_{\text {trailsB }}$, mPACC $_{\text {digit }}$, and CDR-SB, respectively, were identified by both comb-p and DMRcate algorithms; these included DMRs annotated to HOXA4. Furthermore, 5 and 19 DMRs were associated with conversion status in CN and MCl participants, respectively. The most significant DMR was annotated to the AD-associated gene PM20D1 (chr1: 205,818,956 to 205,820,014 [13 probes], Sidak-corrected $P=7.74 \times 10^{-24}$ ), which was associated with both the slope of CDR-SB and the MCl conversion status.
\end{abstract}

Conclusion: Candidate $\mathrm{CpG}$ sites and regions in peripheral blood were identified as associated with the rate of cognitive decline in participants in the ADNI cohort. While we did not identify a single CpG site with sufficient clinical utility to be used by itself due to the observed effect size, a biosignature composed of DNA methylation changes may have utility as a prognostic biomarker for AD progression.

Keywords: Epigenetics, EWAS, DMP, DMR, Alzheimer's disease

*Correspondence: qli2@its.jnj.com

${ }^{1}$ Neuroscience, Janssen Research and Development, LLC, 1125 Trenton-Harbourton Road, Titusville, NJ 08560, USA

Full list of author information is available at the end of the article

\section{Background}

Nearly all (99.6\%) clinical trials of Alzheimer's disease (AD) that were registered at ClinicalTrials.gov during 2002 to 2012 failed, likely because of inadequate understanding of disease pathways and drug targets and original author(s) and the source, provide a link to the Creative Commons licence, and indicate if changes were made. The images or other third party material in this article are included in the article's Creative Commons licence, unless indicated otherwise in a credit line to the material. If material is not included in the article's Creative Commons licence and your intended use is not permitted by statutory regulation or exceeds the permitted use, you will need to obtain permission directly from the copyright holder. To view a copy of this licence, visit http://creativecommons.org/licenses/by/4.0/. The Creative Commons Public Domain Dedication waiver (http://creativeco mmons.org/publicdomain/zero/1.0/) applies to the data made available in this article, unless otherwise stated in a credit line to the data. 
difficulty in identifying patients at early stages of the disease [1]. A total of 2621 trials for AD have been completed, yet the disease remains incurable [2]. Over the years, the United States Food and Drug Administration (FDA) has approved a limited number of drugs that provide symptomatic relief [3]. Although no curative treatments currently exist for AD, several disease-modifying therapies that aim to slow disease progression, especially during early stage, are under investigation $[4,5]$. As of January 5, 2021, 126 investigative agents were in clinical trials for AD; most of them targeting the biological processes underlying AD to modify the disease [6]. Several biomarkers that may be correlated with disease progression and prognosis are being pursued. A new drug that reduces beta-amyloid $(A \beta)$ plaques and may delay disease progression in patients with $\mathrm{AD}$ recently received an accelerated FDA approval [7].

The Alzheimer's Disease Neuroimaging Initiative (ADNI) is an important data source available to the scientific community that greatly contributes to the understanding of $\mathrm{AD}$ progression [8]. An analysis of cerebrospinal fluid (CSF) samples from 287 participants in the ADNI database over 3 to 6 years identified a 16-peptide signature that could not only differentiate between patients with $\mathrm{AD}$ and cognitively normal $(\mathrm{CN})$ participants but could also predict progression from mild cognitive impairment (MCI) to AD better than the traditional $A \beta$ and tau biomarkers [9]. Similarly, a 24-month single-center imaging study showed that deposition of $A \beta$ and cortical thickness are more sensitive biomarkers of disease progression in early AD compared with neuronal dysfunction [10]. Another recent study of patients from both Japanese and North American ADNI databases, which defined disease progression based on the change in Clinical Dementia Rating Scale Sum of Boxes (CDR$\mathrm{SB})$ scores ( $\geq 1$, progression; $<1$, stable), identified several prognostic factors in early AD: baseline tau protein levels in CSF and scores from Mini-Mental State Examination (MMSE), Functional Activities Questionnaire, and 13-item Alzheimer's Disease Assessment Scale-cognition subscale [11].

Neuroinflammation and activated microglia are also considered to play key roles in $\mathrm{AD}$ progression by interacting with $A \beta$ pathways [12], which may offer additional biomarkers of $\mathrm{AD}$ progression. A recent study showed a correlation between baseline levels of CSF cytokine CCL2 and the rate of cognitive decline in patients with MCI who have CSF biomarkers consistent with AD [13]. Furthermore, machine learning algorithms are being used to predict disease progression. A neural network model based on data from 1737 participants in the ADNI database was effective in predicting $\mathrm{AD}$ progression in a separate set of 110 participants who were $\mathrm{CN}$ or had
MCI at baseline [14]. Other machine learning-based models have employed neuroimaging and noninvasive methods using blood biomarkers to predict $\mathrm{AD}$ progression $[15,16]$.

Despite these promising leads, additional biomarkers such as DNA methylation status may shed light on disease progression and potentially shorten the duration of clinical trials of AD. DNA methylation in peripheral blood has been used as a diagnostic biomarker in other diseases [17-19]. Although studies evaluating epigenetic markers in peripheral blood have shown differential DNA methylation in patients with $\mathrm{MCI}$ or $\mathrm{AD}$ compared with $\mathrm{CN}$ participants, suggesting that a DNA methylationbased biosignature could potentially serve as a surrogate of AD diagnosis [20-23], these studies did not use longitudinal clinical data to assess whether peripheral DNA methylation might be associated with disease progression and prognosis. Peripheral DNA methylation was recently shown to be associated with normal brain aging and cognitive decline (e.g., cognitively impaired [Montreal Cognitive Assessment or MoCA <26]) vs. cognitively normal [MoCA > 26]) [24]. DNA methylation in blood was previously studied in nonconverters versus converters to $A D$ using Infinium ${ }^{\circledR}$ HumanMethylation450K BeadChip [25]; however, to our knowledge, it has not been profiled as a biomarker of $\mathrm{AD}$ progression and prognosis using the higher density Infinium ${ }^{\circledR}$ MethylationEPIC BeadChip.

This study aimed to investigate the relationship between baseline peripheral DNA methylation level and disease progression. The earliest available DNA sample from a participant (baseline) was analyzed to assess whether the epigenetic profile obtained at a single clinical visit is associated with the disease trajectory. Cognitive changes in patients across different stages of the disease continuum are captured using different scales, each presumably most sensitive to change in cognitive function for a specific disease stage. For example, the Alzheimer Disease Cooperative Study-Preclinical Alzheimer Cognitive Composite (PACC) was designed as the primary outcome measure of cognitive decline in patients at the asymptomatic phase of $\mathrm{AD}$ [26]. Using the PACC score at 24 months to measure cognitive decline in study participants with preclinical AD, Donohue et al. previously showed that $A \beta$-positive participants had significantly more decline than A $\beta$-negative participants [26]. Accordingly, the PACC, adapted for the available tests in ADNI [27], was used in the present study to assess cognitive decline in participants who were $\mathrm{CN}$ at baseline. The CDR-SB scale, which provides more detailed staging information compared with CDR global score for patients with MCI [28], was used to assess cognitive decline in patients with $\mathrm{MCI}$ at baseline to optimize the sensitivity to change in this population $[29,30]$. Lower PACC scores 


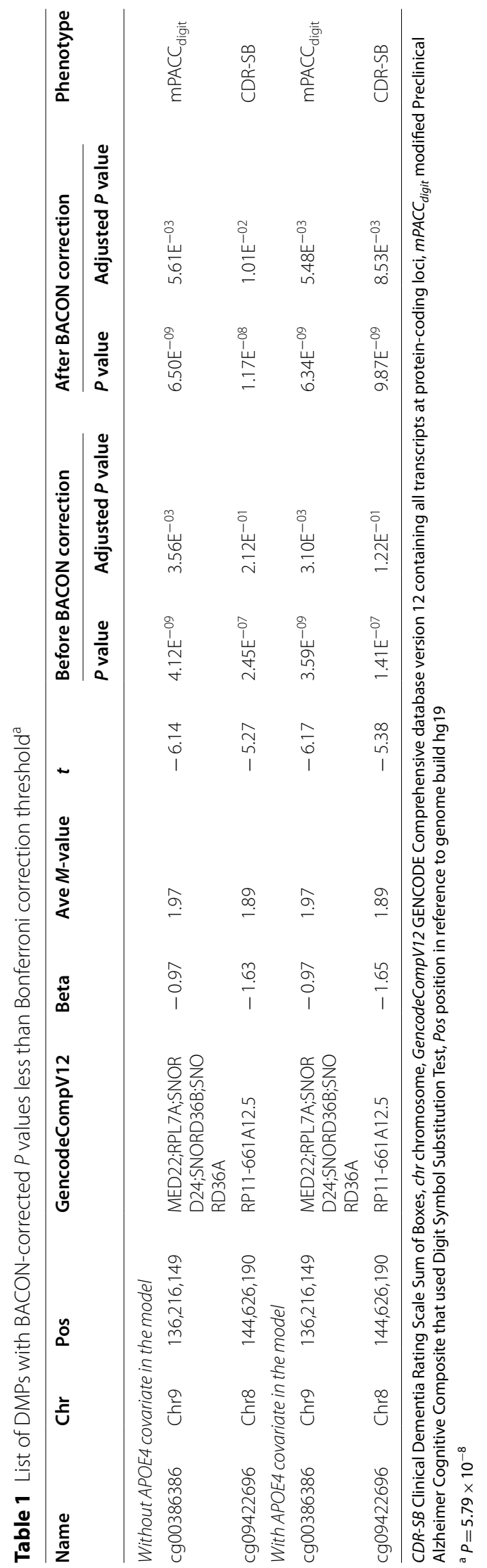


and higher CDR-SB scores represent poorer cognitive performance. In addition, dichotomized endpoints were used to assess conversion status in both $\mathrm{CN}$ participants and patients with $\mathrm{MCI}$.

\section{Results}

\section{Disease progression}

The association of DNA methylation with disease progression was analyzed in 2 diagnosis groups: $\mathrm{CN}$ and MCI. Among 202 participants in the $\mathrm{CN}$ group, 56 (27.7\%) converted from $\mathrm{CN}$ at baseline to $\mathrm{MCI}$ (converters) and 146 (72.3\%) did not (nonconverters), at up to 8 years since DNA sampling. The mean (SD) follow-up period since DNA sampling in the CN group was 54.1 (21.5) months for converters and 61.2 (19.4) months for nonconverters. Among 317 patients in the MCI group, 115 (36.3\%) converted from MCI to AD and 202 (63.7\%) were nonconverters. The mean (SD) follow-up time since DNA sampling in the MCI group was 43.6 (20.9) months for converters and 57.2 (18.1) months for nonconverters.

\section{Differentially methylated positions in peripheral blood}

Two Cytosine-phosphate-guanine (CpG) sites were associated with the rate of cognitive decline in $\mathrm{CN}$ participants and patients with MCI at a level surpassing Bonferroni correction threshold of $P=5.79 \times 10^{-8}$ (Table 1). After BACON correction [31], all lambda values were < 1.04 (Additional file 1: Fig. 1 [Q-Q plots]; Fig. 2 [Manhattan plots]). In $\mathrm{CN}$ participants, cg00386386 was associated with the rate of cognitive decline as measured by the slope of modified PACC (mPACC) that used $\mathrm{mPACC}_{\text {digit }}\left(P=6.50 \times 10^{-9}\right) \quad$ (Table 1; Fig. 1A). In patients with MCI, cg09422696 annotated to RP11-661A12.5 was associated with the rate of cognitive decline as measured by the slope of CDR-SB $\left(P=1.17 \times 10^{-8}\right)$ (Fig. 1B, Table 1). Single nucleotide polymorphism (SNP) rs2382954 was correlated with DNA methylation level at cg09422696 $\left(\mathrm{r}=-0.98, P<2.2 \mathrm{e}^{-16}\right)$ and hence a methylation quantitative trait locus (mQTL) in cis (Fig. 1C), as reported previously [32]. The SNP rs2382954 was also associated with the slope of CDRSB $\left(P=8.12 \times 10^{-5}\right)$. When adjusted for rs2382954, the association between cg09422696 methylation level and the slope of CDR-SB failed to meet the significance threshold $(P>0.05)$, suggesting that the association was driven solely by rs2382954. A list of $P$ values suggestive of associations of other CpG sites with the rate of cognitive decline $\left(P<1 \times 10^{-5}\right)$ is available in Additional file 2: Table 1 . No CpG probe was significantly associated with disease conversion status (ie, $\mathrm{CN}$ to $\mathrm{MCI}$ or $\mathrm{MCI}$ to $\mathrm{AD}$ ) (Additional file 1: Figs. 1C, E, 2C, and E) passing Bonferroni correction threshold.
Using all baseline study samples from the ADNI cohort, mPACC $_{\text {digit }}$ was negatively correlated with CDR-SB $\left(r=-0.787, P<2.2 \mathrm{e}^{-16}\right)$ and highly correlated with mPACC $_{\text {trailsB }}\left(r=0.980 ; \quad P<2.2 \mathrm{e}^{-16}\right)$ (Additional file 1: Fig. 3). For participants who were $\mathrm{CN}$ at baseline, the correlation between $\mathrm{mPACC}$ digit and CDR-SB was reduced $(r=-0.128 ; P=0.004)$, and the dynamic range for $\mathrm{mPACC}_{\text {digit }}$ was wider than that for CDR-SB (0-1) (Additional file 1: Fig. 4A), suggesting that $\mathrm{PPACC}_{\text {digit }}$ measures the cognitive change in an asymptomatic population. Despite the significant but attenuated correlation between mPACC $_{\text {digit }}$ and CDR-SB among baseline measurements from participants who were $\mathrm{MCI}$ at baseline $\left(r=-0.324, P<2.2 \mathrm{e}^{-16}\right.$, Additional file 1: Fig. $\left.4 \mathrm{~B}\right)$, the range of $\mathrm{mPACC}_{\text {digit }}$ did not seem to vary much for participants with CDR-SB ranging from 0 to 5.5, supporting the choice of using CDR-SB to capture the change in cognitive function among patients who were $\mathrm{MCI}$ at baseline. Additional file 1: Fig. 4C shows correlation between $\mathrm{mPACC}_{\text {digit }}$ and CDR-SB for participants who were either $\mathrm{CN}$ or MCI at baseline $\left(r=-0.637 ; P<2.2 \mathrm{e}^{-16}\right)$.

The effect sizes for the association between the slope of mPACC $_{\text {trailsB }}$ and methylation level of CpG probes were correlated with the effect sizes for $\mathrm{mPACC}_{\text {digit }}(r=0.997$, $P<2.2 \mathrm{e}^{-16}$ for probes with $P_{\mathrm{DMP}}<1 \times 10^{-5}$ in either analysis). The effect sizes for the slope of $\mathrm{mPACC}_{\text {trailsB }}$ were negatively correlated with the effect sizes for the conversion status in CN participants $\left(r=-0.660, P=5.545 \mathrm{e}^{-8}\right.$ for probes with $P<1 \times 10^{-5}$ in either analysis, Fig. $2 \mathrm{~A}$ ) but not correlated with the effect sizes for the slope of CDR-SB $(r=0.020, P=0.860)$ or the effect sizes for the conversion status in patients with MCI $(r=-0.024$, $P=0.868$ ) for the probes with $P<1 \times 10^{-5}$ in either analysis. In contrast, the effect sizes for the slope of CDRSB were correlated with those for conversion status in patients with MCI $\left(r=0.866, P<2.2 \mathrm{e}^{-16}\right.$ for the probes with $P<1 \times 10^{-5}$ in either analysis; Fig. $2 \mathrm{~B}$ ), suggesting that the effect sizes from the continuous endpoints are concordant with those from the paired dichotomized endpoints, but the effect sizes in the two populations (CN vs. MCI at baseline) are quite far apart.

Gene set enrichment analysis (GSEA) using methylGSA revealed enrichment of mitogen-activated protein kinase signaling pathway $(P=0.002$, adjusted $P=0.05)$ and $\mathrm{Fc}$ epsilon RI signaling pathway $(P=0.01$, adjusted $P=0.14)$ for the rate of cognitive decline as measured by the slope of $\mathrm{mPACC}_{\text {trailsB }}$ (Additional file 2: Table 2A). Overrepresentation analysis of $\mathrm{CpG}$ probes with nominal $P<0.01$ revealed a common theme of enrichment of genes involved in neurogenesis for the rate of cognitive decline as measured by the slopes of $\mathrm{mPACC}_{\text {trailsB }}$ (Additional file 2: Table $2 \mathrm{~A}$ ), $\mathrm{mPACC}_{\text {digit }}$ (Additional file 2: Table 2B), CDR-SB $\left(P=7.00 \times 10^{-6}, \quad\right.$ FDR adjusted $P=0.006$, 

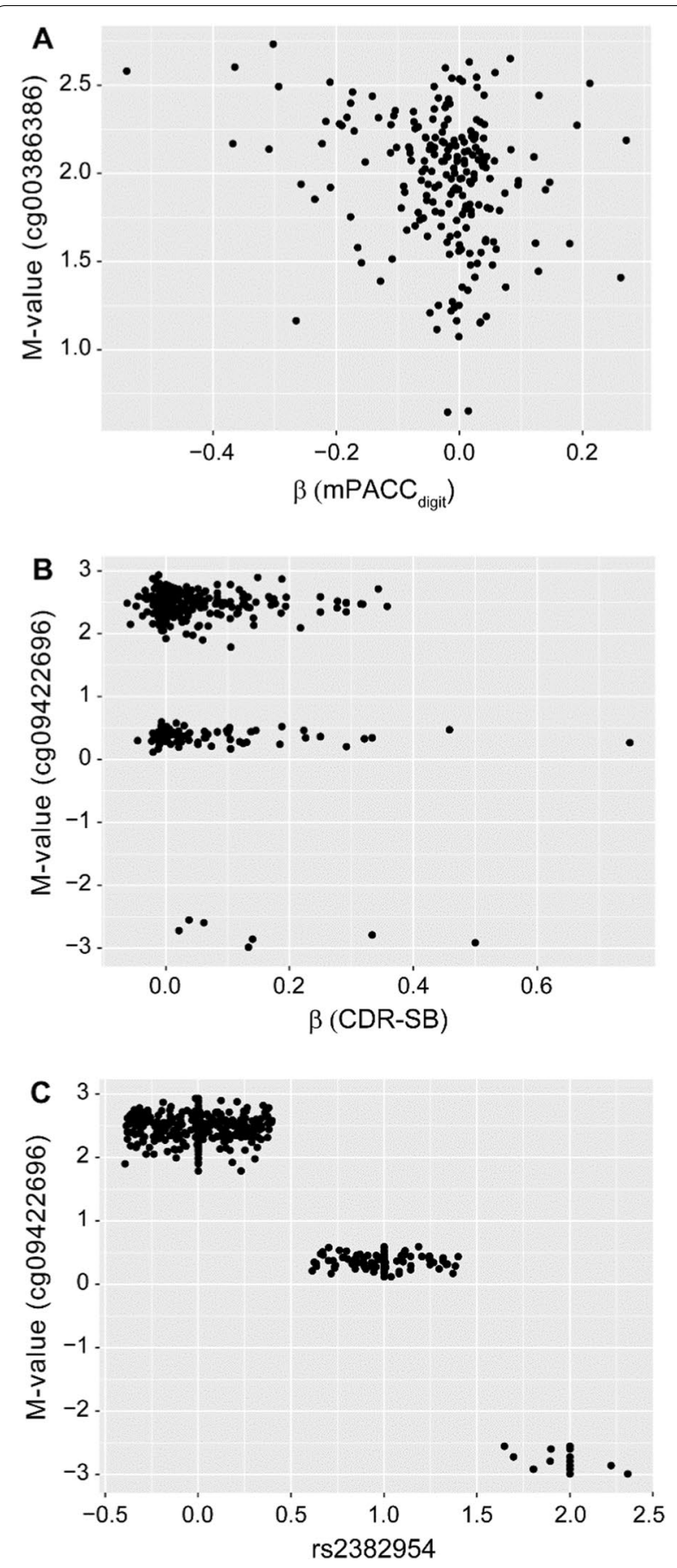

Fig. 1 Association of $\mathrm{CpG}$ sites with rate of cognitive decline. A cg00386386 was associated with the slope of PACC $_{\text {digit }}$ in CN participants. B cg09422696 was associated with the slope of CDR-SB in patients with MCl. C rs2382954-cg09422696 mQTL. Note that jitter was introduced for the $x$-axis. CDR-SB Clinical Dementia Rating Scale Sum of Boxes, CN cognitively normal, $M C$ l mild cognitive impairment, mPACC digit modified Preclinical Alzheimer Cognitive Composite that used Digit Symbol Substitution Test, $m Q T L$ methylation quantitative trait locus
Additional file 2: Table 2C), and for conversion status in the CN participants (Additional file 2: Table 2D). Gene sets involved in cognition were also nominally enriched for the rate of cognitive decline as measured by the slope of $\mathrm{mPACC}_{\text {trailsB }}(P=0.0007$, adjusted $P=0.07$, Additional file 2: Table 2A) and conversion status in patients with MCI $(P=0.0001$, Additional file 2: Table 2E).

We compared our results with the analyses of followup samples from 2 studies-AddNeuroMed (comparing MCI to AD converters with nonconverters) and MiddleAged Danish Twin (MADT) studies-which evaluated the relationship between epigenetic profiles and cognitive changes over 10 years [33,34]. The effect size from the top 1000 DMPs from the AddNeuroMed study [33] was weakly correlated with the effect size from the MCI to AD conversion status in this study $(r=0.1, P=0.002$, Additional file 1: Fig. 5). There are $22 \mathrm{CpG}$ sites associated with disease progression with consistent directionality and nominal $P<0.05$ between this study and AddNeuroMed study (Additional file 2: Table 3), including cg20152430 $(P=0.003$ in AddNeuroMed; $P=0.003$ in this study) that annotated to HOXB3 and cg12559197 $(P=0.001$ in the AddNeuroMed study; $P=0.0008$ in this study) that annotated to $P D E 8 B$. In the MADT study [34], only 12 probes were reported to be associated with cognitive change over 10 years, among which cg27630540 annotated to KIAA1530 $\left(\beta=-0.01, P=8.25 \times 10^{-6}\right.$ in the MADT study) trended in the same direction $(\beta=-0.39, P=0.06)$ as the slope of $\mathrm{mPACC}_{\text {trailsB }}$ analysis in this study, and cg13630845 annotated to SLC35E1 $\left(\beta=-0.0247, P=7.73 \times 10^{-6}\right.$ in the MADT study) trended in the same direction as observed in the $\mathrm{CN}$ to MCI converter versus nonconverter analysis $(\beta=0.04$, $P=0.1$ ).

There was no correlation at the CpG level between the effect sizes for the slope of either $\mathrm{MPACC}_{\text {trailsB }}$ or CDRSB and the effect size for the $220 \mathrm{CpG}$ sites associated with Braak stage across cortex in the brain previously identified in an epigenome-wide association meta-analysis study (EWAS) [35] (Additional file 1: Figs. 6A, B). Similarly, there was no correlation between the effect sizes for the clinical diagnosis (AD vs. $\mathrm{CN}$ ) in blood published previously [20] and the effect size for the $220 \mathrm{CpG}$ sites across cortex in the EWAS meta-analysis (Additional file 1: Fig. 6C), suggesting a generally low concordance between peripheral blood and brain, although the compared phenotypes are not identical, and the blood and brain data were not from the same individuals. In addition, there was no correlation at the $\mathrm{CpG}$ level between the effect sizes for the slope of either mPACC trailsB or CDR-SB and the effect size for AD diagnosis in blood from the same ADNI cohort [20] (Additional file 1: Fig. 7A and B). 

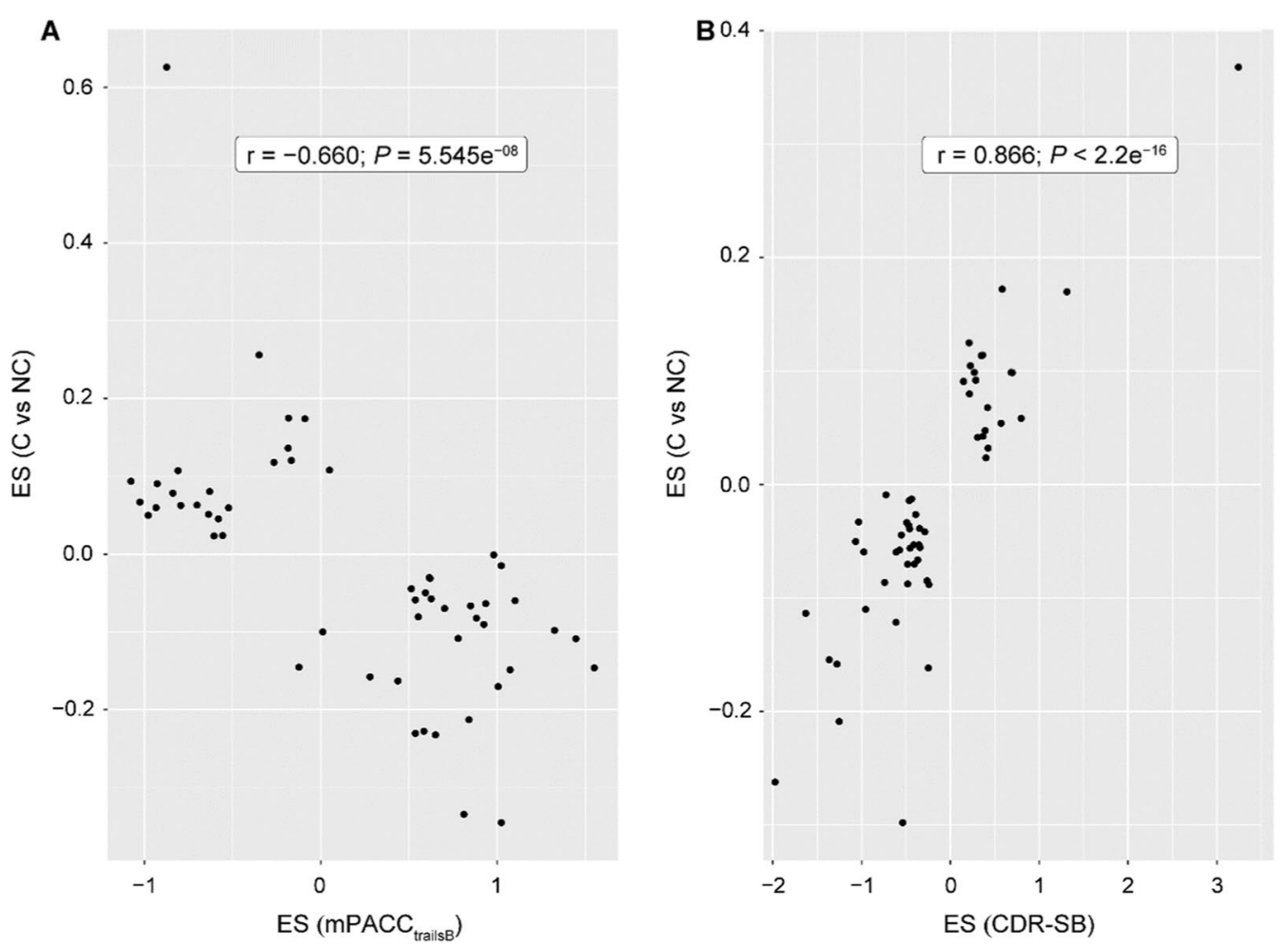

Fig. 2 The correlation of the effect size from DMP analysis for the slope of $\mathrm{MPACC}_{\text {trailsB }}$ conversion status in CN participants (A) and the slope of CDR-SB vs. conversion status in patients with $\mathrm{MCl}(\mathbf{B})$. Only the probes with $P<1 \times 10^{-5}$ in either analysis are plotted. CDR-SB Clinical Dementia Rating Scale Sum of Boxes, CN cognitively normal, DMP differentially methylated positions, $M C /$ mild cognitive impairment, mPACC $_{\text {trails } B}$ modified Preclinical Alzheimer Cognitive Composite that used Trail-Making Test Part B

\section{Differentially methylated region (DMR) analysis}

DMR analysis enabled identification of regions in the genome consisting of $\geq 3$ probes. Overall, comb-p algorithm identified 70 and 76 DMRs to be significantly associated with the rate of cognitive decline as measured by the slopes of $\mathrm{mPACC}_{\text {trailsB }}$ and mPACC digit, respectively, in participants who were $\mathrm{CN}$ at baseline (Additional file 2: Tables 4A and B). Among these, 47 DMRs were common between the two endpoints, including a DMR annotated to HOXA4 (chr7: $27,170,241$ to $27,171,051$ [14 probes], Sidakcorrected $P=3.26 \times 10^{-6}$ ) associated with the slope of mPACC $_{\text {trailsB }}$ (Additional file 1: Fig. 8A) and an overlapping DMR annotated to HOXA4 (chr7: $27,169,957$ to $27,171,154$ [16 probes], Sidak-corrected $P=1.24 \times 10^{-10}$ ) associated with the slope of mPACC digit (Additional file 1: Fig. 8B). In blood, the increased levels of methylation or probes in the DMR annotated to HOXA4 were associated with slower rate of cognitive decline (Additional file 1: Fig. 8A). In addition, 5 DMRs annotated to the protocadherin gamma gene cluster were also identified as associated with the slope of mPACC $_{\text {digit }}$, including DMRs annotated to PCDHGA4 (chr5: $140,734,648$ to $140,735,027$ [4 probes], Sidakcorrected $P=0.002$ ), PCDHGA10 (chr5: 140,794,664 to $140,794,993$ [3 probes], Sidak-corrected $P=0.007$ ), PCDHGB7 (chr5: $140,796,045$ to $140,796,312$ [3 probes], Sidak-corrected $P=0.008), \quad$ PCDHGA11 (chr5: $140,802,432$ to $140,802,831$ [4 probes], Sidak-corrected $P=0.002$ ), and PCDHGA12 (chr5: $140,810,051$ to $140,810,433$ [9 probes], Sidak-corrected $\left.P=1.75 \times 10^{-5}\right)$. DMRcate detected fewer DMRs $(24$ and 16 for $\mathrm{mPACC}_{\text {trailsB }}$ and $\mathrm{mPACC} \mathrm{Cigit}_{\text {dit }}$, respectively), among which 19 and 13 were also detected by comb-p. The DMRs annotated to HOXA4 and PCDHGA12 were detected by both methods.

A total of 102 and 5 DMRs, detected by comb-p and DMRcate, respectively (all 5 detected by DMRcate were also detected by comb-p), were significantly associated with the rate of cognitive decline as measured by slope of CDR-SB in patients who had MCI at baseline (Sidak-corrected $P<0.05$, Additional file 2: Table 4C). This included a DMR annotated to HOXB6 (detected by comb-p only, chr17: 46,681,111-46,681,550 [7 probes], Sidak-corrected $P=0.0002$ ) and a DMR annotated to HOXB9 (detected by comb-p only, chr17: 46,698,598-46,699,155 
[7 probes], Sidak-corrected $P=0.002$ ); however, one DMR was hypermethylated and the other was hypomethylated (Additional file 1: Figs. 8C and D).

For the dichotomized conversion status association, 75 and 123 DMRs were identified by comb-p in study participants who were $\mathrm{CN}$ or had MCI at baseline, respectively (Additional file 2: Tables $4 \mathrm{D}$ and $4 \mathrm{E}$ ), replicating two previously reported DMRs (KCNAB3 and GABBR1) associated with MCI conversion status from the AddNeuroMed study [33]. For the probe annotated to GABBR1 among the top 1000 DMPs associated with $\mathrm{MCI}$ to AD conversion status in the AddNeuroMed study (cg06512249, $\beta=-0.03, P=0.003$ ), the direction was consistent with multiple probes in the same region from this study (cg03316098, $\beta=-0.05, P=0.01 ; \operatorname{cg} 10234998$, $\beta=-0.10, P=0.005$; cg12061917, $\beta=-0.07, P=0.001$; cg21481950, $\beta=-0.06 \quad P=0.007)$ even though cg06512249 did not reach the nominally significant threshold in this study $(P>0.05)$ (Additional file 1: Fig. $8 \mathrm{E})$. The probe from the $K C N A B 3$ was not among the top 1000 DMPs associated with MCI to AD conversion status in the AddNeuroMed study, so the directionality could not be confirmed in this study. The most significant DMR identified in this study that was associated with conversion status in patients with MCI was annotated to PM20D1 (chr1: 205,818,956 to 205,820,014 [13 probes],

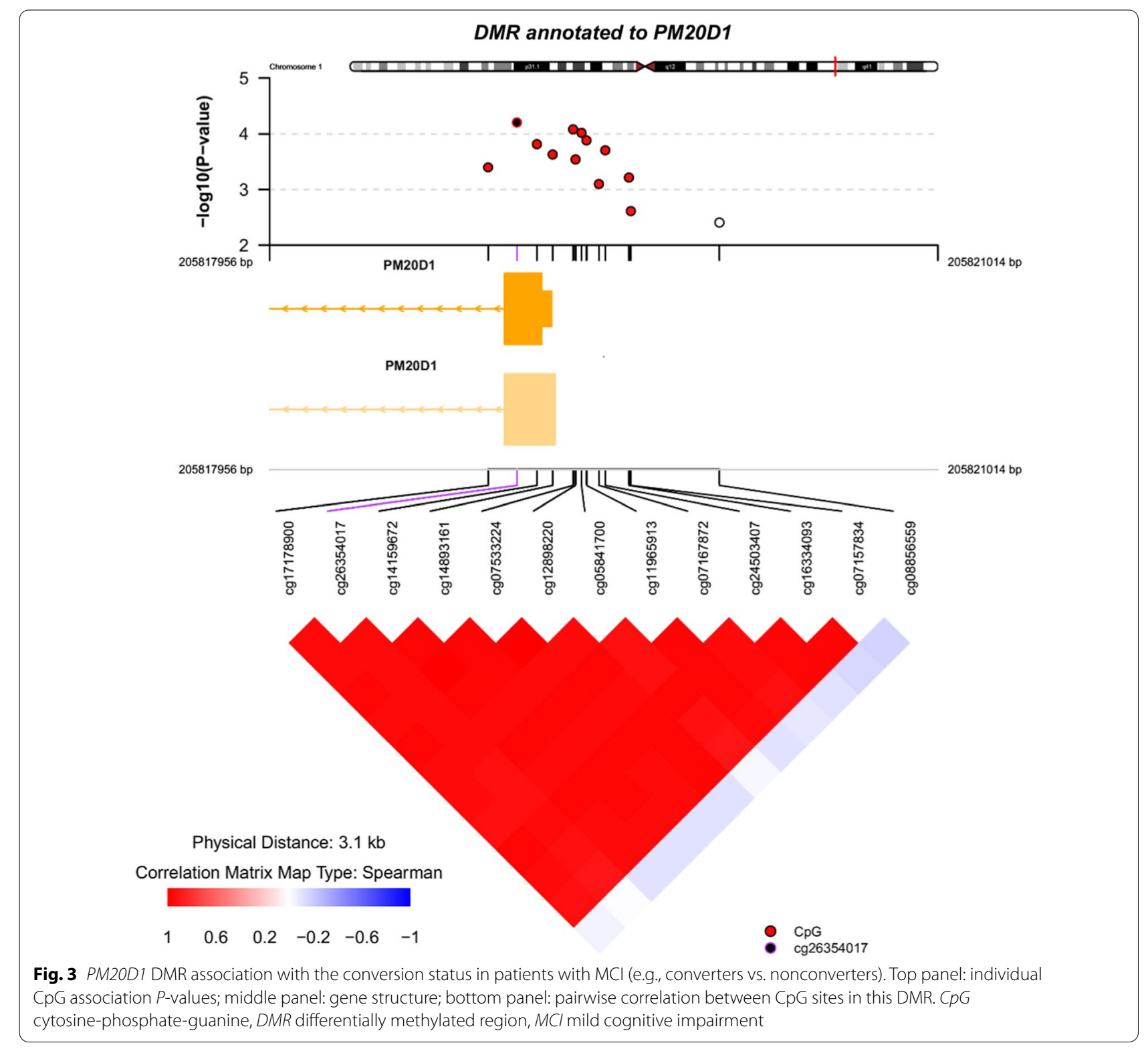


Sidak-corrected $P=7.74 \times 10^{-24}$ ) (Fig. 3). A similar DMR annotated to PM20D1 (chr1: 205,818,956 to 205,819,609 [12 probes], Sidak-corrected $P=7.00 \times 10^{-5}$ ) was also associated with the rate of cognitive decline as measured by the slope of CDR-SB in patients with MCI. In addition, a DMR annotated to DUSP22 (chr6: 291,634 to 292,823 [10 probes], Sidak-corrected $P=8.09 \times 10^{-14}$, Additional file 1: Fig. 8F) was associated with conversion status in $\mathrm{CN}$ participants. DMRcate identified fewer DMRs (6 and 21 in study participants who were $\mathrm{CN}$ or had MCI at baseline, respectively), among which 5 and 19, respectively, overlapped with those identified by comb-p. The DMRs annotated to PM2OD1 and DUSP22 were detected by both DMR methods.

Although the DMRs annotated to OXT and LDLRAD4 associated with conversion status to $\mathrm{AD}$ in this study and the German Study on Aging, Cognition and Dementia in Primary Care Patients (AgeCoDe) [25], the probes in this study were hypomethylated among converters in contrast to the reported hypermethylation in blood in the AgeCoDe cohort study. Another DMR annotated to PRRT1 that associated with conversion status to AD in the AgeCoDe study, was also associated with both the slope of mPACC $_{\text {trailsB }}$ (detected by both comb-p and DMRcate) and CDR-SB in this study but in opposite direction.

Multiple other DMRs were detected: ZFP57 (identified by both comb-p and DMRcate); LMTK3 (Additional file 1: Fig. 8G) and FBXO44 (identified by comb-p only) for the rate of cognitive decline as measured by the slope of CDR-SB in patients with MCI (Additional file 2: Table 4C); LOC105375131 and GAL3ST2 (both identified by comb-p alone) for conversion status in $\mathrm{CN}$ participants (Additional file 2: Table 4D); and FGFR2 (identified by both comb-p and DMRcate) for conversion status in patients with MCI (Additional file 2: Table 4E).

Furthermore, DMRs annotated to MORN4, AIRE, $L Y 6 G 5 C$, and PRRT1 were identified for both rates of cognitive decline as measured by the slopes of mPACC digit $/ \mathrm{mPACC}_{\text {trailsB }}$ and CDR-SB. In addition, DMRs annotated to FOXK1, $A C Y 3$, and $Z B E D 9$ were identified for conversion status in both subjects with $\mathrm{CN}$ and $\mathrm{MCI}$ at baseline. With the exception of the DMR annotated to $A C Y 3$ that was detected by both comb-p and DMRcate, the rest of DMRs were detected by comb-p alone. The overlap between DMRs association in the $\mathrm{CN}$ and MCI populations appears to be small, consistent with the low correlation of effect size in DMP analysis between the two populations. In contrast, there were 47 overlapped DMRs between the rate of cognitive endpoints defined by the slope of mPACC digit versus mPACC $\mathrm{trailsB}_{\text {. }}$

Genes involved in neuronal postsynaptic density were enriched among DMRs associated with the rate of cognitive decline as measured by $\mathrm{mPACC}_{\text {trailsB}}$, and this enrichment was driven by DMRs annotated to BNIP3, NTRK2, and BAIAP2 (Additional file 2: Table 5A). Similarly, genes involved in regulation of postsynaptic density assembly or organization were enriched among DMRs associated with conversion status in $\mathrm{CN}$ participants (Additional file 2: Table 5B). Notably, genes encoding neural cadherin-like cell adhesion proteins are highly enriched among the DMRs associated with the slope of $\mathrm{mPACC}_{\text {digit }}$ (Additional file 2: Table 5C). Additional ontology terms that were enriched are shown in Additional file 2: Tables 5D and E. Furthermore, genes with literature evidence linking to neurogenesis, cognition, amyloid, and tau pathology are shown in Fig. 4 and Additional file 2: Table 6.

\section{Discussion}

We assessed the association between disease progression and DNA methylation in $\mathrm{CN}$ participants and patients with MCI. Approximately $30 \%$ of CN participants converted to $\mathrm{MCI}$ and approximately $36 \%$ of patients with MCI converted to AD in a mean follow-up period of 54.1 and 43.6 months, respectively. Two differentially methylated positions (DMPs) were significantly associated with cognitive decline at a level surpassing Bonferroni correction threshold: cg00386386 was associated with the slope of mPACC $_{\text {digit }}$ in CN participants, and cg09422696 was associated with the slope of CDR-SB in patients with MCI. When adjusted for rs2382954, the association between cg09422696 methylation level and the slope of CDR-SB was no longer statistically significant, suggesting that the association was driven solely by rs2382954. No CpG sites were identified that were significantly associated with diagnosis conversion status.

In this study, we found several DMRs previously implicated in $\mathrm{AD}$, including those annotated to HOXA4, $H O X B 6 / H O X B 9$ that were associated with the rate of cognitive decline as measured by the slopes of mPACC trailsB $/ \mathrm{mPACC}_{\text {digit }}$ and CDR-SB, respectively; and those annotated to DUSP22 and PM2OD1 that were associated with conversion status in $\mathrm{CN}$ and MCI participants, respectively. The DMRs annotated to HOXA4 and $H O X B 6 / H O X B 9$ gene clusters have been previously shown to be associated with tau pathology and/or neurogenesis [33, 35-42]. The decreased levels of methylation for probes in the DMR annotated to HOXA4 were associated with faster rate of cognitive decline; in contrast, hypermethylation was reported for probes annotated to the HOXA gene cluster in the brain in patients with $\mathrm{AD}$ [24, 35, 40-42]. A cell-type specific EWAS suggested that the hypermethylation was derived from neuronal cells [43]. In the present study, the hypermethylation of probes in the DMRs annotated to HOXB6 was associated with faster rate of cognitive decline, whereas 


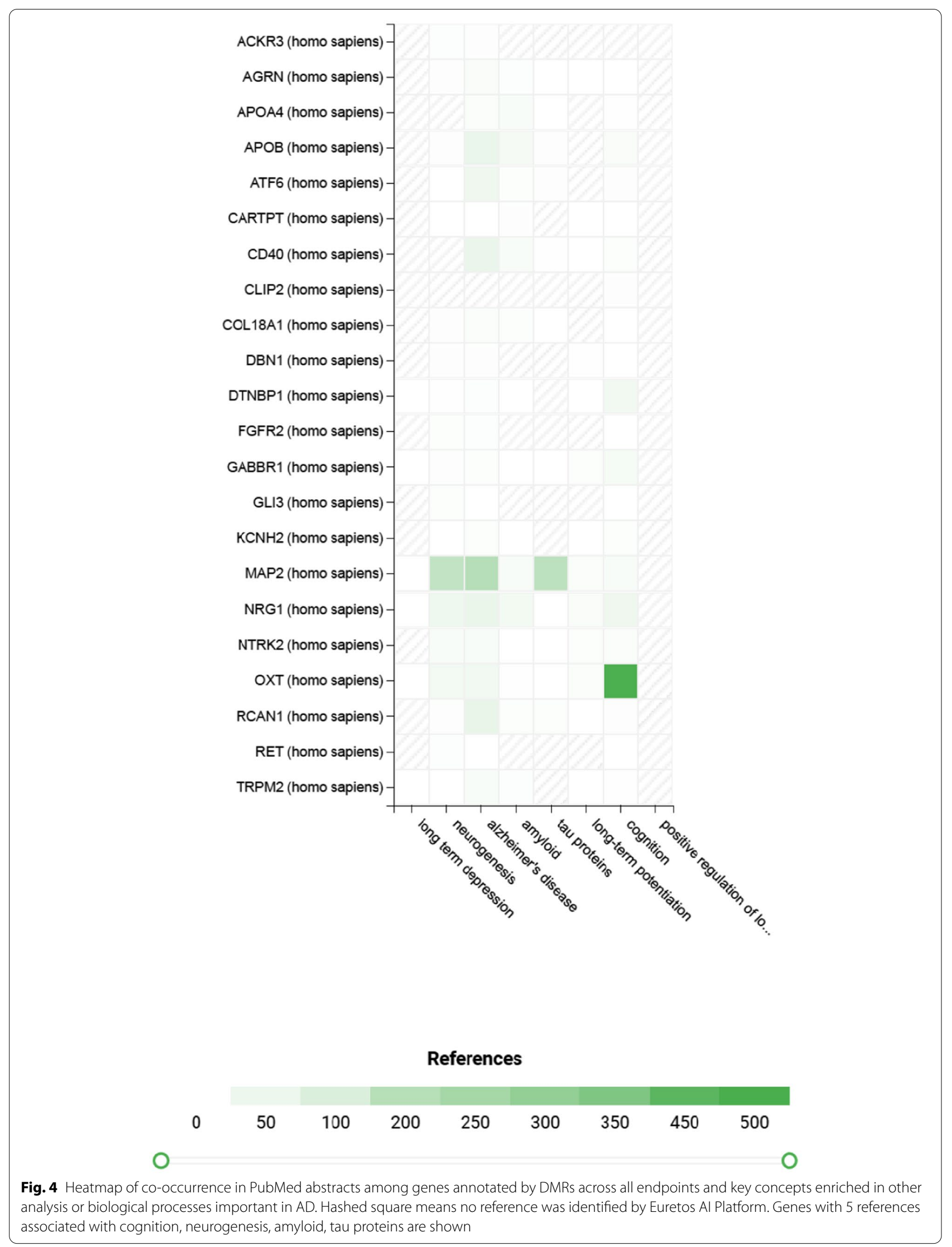


the hypermethylation of probes in the DMRs annotated to $H O X B 9$ was associated with slower rate of cognitive decline. The hypermethylation in the HOXB gene cluster and $H O X B 6$ gene were previously reported in brain and blood, respectively [33, 35, 41]; in contrast, expression of HOXB9 was reported to be increased in the brain tissue from patients with Huntington disease [44]. The effect sizes for the association between $\mathrm{CpG}$ and the slope of neither $\mathrm{mPACC}_{\text {trailsB }}$ nor CDR-SB were correlated with the effect sizes for the $220 \mathrm{CpG}$ sites across cortex previously identified [35]. DMR annotated to $A P O B$ associated with $\mathrm{CN}$ to $\mathrm{MCI}$ conversion status (called by both comb-p and DMRcate) was also implicated in neuronal survival and maintenance [45] and previously identified as a DMR in hippocampus in AD patients [24].

The most significant DMR was identified in the ADassociated gene PM20D1 [46, 47], which was associated with both the slope of CDR-SB (called by comb-p) and the MCI to AD conversion status (called by both comb$\mathrm{p}$ and DMRcate). While variants associated with $\mathrm{AD}$ have been characterized as both meQTL and eQTL for $P M 20 D 1$, the differential methylation of $P M 20 D 1$ promoters in human samples of AD and differential expression of PM20D1 in mouse models and human samples of AD were reported [47]. PM20D1 expression is upregulated by $A D$-related stressors, amyloid- $\beta$ and reactive oxygen species, and overexpression of PM20D1 was neuroprotective in cell and primary cultures [46]. DUSP22 promoter hypermethylation and mRNA downregulation in the hippocampus of $\mathrm{AD}$ patients were reported previously [48]. Further, functional experiments demonstrated that shRNA silencing of DUSP22 caused the loss of tau phosphorylation at Thr231, while overexpression of DUSP22 increased Thr231 tau phosphorylation via a mechanism mediated by PKA and p38 [48]. Phosphorylation at Thr231 is one of the first phosphorylation events of tau protein in the AD disease process [49]. The hypomethylation of probes in the DMRs annotated to DUSP22 was associated with $\mathrm{CN}$ to $\mathrm{MCI}$ conversion status. DUSP22 also affects CREB activity via PKA; CREB activity, which has been shown to be important for neuronal survival, axonal growth, and synaptic function $[50,51]$, is altered in AD. A DMR annotated to CREB5 and NTRK2 was also associated with the slope of $\mathrm{mPACC}_{\text {trailsB }}$ (Additional file 2: Table 4A). These genes and PTH2R, SALL3, and HOXA4 (DMRs annotated to these genes were also identified in the slope of $\mathrm{MPACC}_{\text {trailsB }}$ analysis) all harbor CCAAT/enhancer-binding protein (C/EBP) gamma binding motif, and genes with one or more occurrence of the transcription factor binding site V\$CEBPGAMMA Q6 [52] are enriched (Additional file 2: Table 6A). PTH2R was identified as a hub gene in a weighted co-expression network analysis using transcriptomic data from the frontal lobe and temporal cortex in patients with vascular dementia or $\mathrm{AD}$ [53]. A decrease in dendritic spine numbers and an imbalance between long-term potentiation and depression caused by oligomeric $A \beta$, can lead to impaired synaptic transmission [51]. Furthermore, through its binding to p75 neurotrophin receptor $\left(\mathrm{p} 75^{\mathrm{NTR}}\right)$ and brain-derived neurotrophic factor receptor (encoded by NTRK2), A $\beta$ can further worsen the situation [51]. Levels of N-cadherin, a synaptic adhesion molecule, are elevated in the plasma, brain, and CSF of patients with AD [54]. Interestingly, the DMRs annotated to $\mathrm{N}$-cadherin-like gene cluster were associated with rate of cognitive decline (Additional file 2: Tables 4A and B). Taken together, the DMRs uncovered in this study appear to implicate genes involved in synaptic plasticity, $A \beta /$ tau pathology, and pathways mediated by DUSP22 and PKA/ MAPK kinase that affect CREB and C/EBPY activities. Even though the same DMRs were identified in brain and blood, the change of methylation pattern appears complex, and the directionality of methylation change was often contradictory between brain and blood.

Interestingly, the DMR annotated to $A C Y 3$ was associated with both the slope of $\mathrm{MPACC}_{\text {trailsB }}$ and MCI to $\mathrm{AD}$ conversion status (called by both comb-p and DMRcate); it was also associated with the slope of $\mathrm{mPACC}_{\text {digit }}$ and $\mathrm{CN}$ to $\mathrm{MCI}$ conversion status (called by comb-p alone), together representing a mechanism relevant to disease progression in both stages ( $\mathrm{CN}$ and $\mathrm{MCI}) . A C Y 3$ was identified as a gene associated with a microglial gene expression profile [55], which was differentially expressed in P301L-tau transgene model [56]. The single cell RNASeq profile confirmed the microglia expression specificity (Additional file 1: Fig. 9A). The role of microglia in $\mathrm{AD}$ was recently underscored in a recent meta-analysis of genome-wide association studies [57, 58]. DUSP22 (Additional file 1: Fig. 9B) and RPL37 (Additional file 2: Tables 4A, 4B; Additional file 1: Fig. 9C) associated with rate of cognitive decline as measured by the slope of mPACC, and AMPD3 (Additional file 2: Tables 4C, 4E; Additional file 1: Fig. 9D) associated with the slope of CDR-SB and MCI to AD conversion status are also abundantly expressed in microglia. Astrocytes play important roles in CNS, including defense against oxidative stress, mitochondria biogenesis, tissue repair and neurogenesis [59]. GABBR1 (Additional file 1: Fig. 9E), ATP6VOE2 (Additional file 2: Tables 1, 4A; Additional file 1: Fig. 9F), NWD1 (Additional file 2: Table 4D; Additional file 1: Fig. 9G), and FGFR2 (Additional file 2: Table 4E; Additional file 1: Fig. $9 \mathrm{H}$ ) are abundantly expressed in astrocytes. Both GABBR1 and FGFR2 are implicated in neurogenesis, learning, and memory [60, 61].

Multiple other DMRs, including FGFR2 for conversion status in patients with $\mathrm{MCI}$, were detected. The 
FGF7/FGFR2/PI3K/Akt pathway has been implicated in microRNA-107-induced increased cell proliferation and reduced cell inflammation and apoptosis in an in vitro model of AD [62]. A DMR annotated to LMTK3 was associated with the rate of cognitive decline as measured by the slope of CDR-SB in patients with $\mathrm{MCI}$, and hypomethylation was associated with faster rate of cognitive decline. Probes annotated to LMTK3 have been reported to be differentially methylated in the cortex in patients with Down syndrome [63] and in both the dorsal motor nucleus of the vagus and the substantia nigra in patients with Parkinson disease [64]. In Tg4-42 mouse, which expresses $\mathrm{N}$-truncated $\mathrm{A} \beta_{4-42}$ and exhibits neuronal loss and behavioral deficits but no plaque formation, the LMTK3 transcript was reported to be differentially downregulated in aged animals [65]. A related family member, $L M T K 2$, which is considered to play a role in neurodegeneration possibly via mechanisms such as tau hyperphosphorylation, enhanced apoptosis, and disrupted axonal transport, was similarly downregulated in an AD animal model [66].

This study has some limitations. Although we replicated the GABBR1 DMR association with cognitive change from the AddNeuroMed study [33], the overall effect size correlation was weak. This may be related to a relatively small sample size in the compared study (38 MCI to AD converters and 67 nonconverters). In addition, the phenotype of conversion in this study was not limited to a one-year follow-up period, although the converters and nonconverters had generally similar follow-up periods, with a slightly longer follow-up for nonconverters. Comparison with the MADT study was limited to 12 reported DMPs with $P<1 \times 10^{-5}$. In addition, the study designs were different (monozygotic twin vs. population study), and the samples were different (the follow-up sample in the MADT study associated with the cognitive change in the preceding 10 years vs. baseline sample in this study associated with the subsequent cognitive change in approximately 5 years). In the present study, the rate of cognitive decline was computed using a linear model (robust regression), which is different from the nonlinear trajectory of cognitive decline previously proposed in a hypothetical model [67]. Even though the motivation for this study was to identify biomarkers for disease progression, we did not find any individual CpG that crossed study-wide significance threshold and had effect size large enough to potentially serve as a prognostic biomarker. A panel of CpGs predictive of disease progression at the individual level cannot be ruled out completely; however, building a generalizable predictive model based on a biosignature would require identification of a set of reliable CpGs associated with disease progression. The sample size in this study for each group was modest after applying the patient population selection criteria, which limits the statistical power for the analysis. The study was conducted on bulk tissue using whole blood or buffy coat. Cell type-specific methylation difference could be obscured in this assay. Replication is needed to confirm the results presented here.

In conclusion, this study replicated multiple DMPs and DMRs and identified additional candidate DMPs and DMRs in peripheral blood that were associated with the rate of cognitive decline in participants in the longitudinal ADNI cohort. The DMRs uncovered in this study implicated genes involved in synaptic plasticity and $A \beta /$ tau pathology and pathways mediated by DUSP22 and PKA/MAPK kinase and impacting CREB and C/EBPY activities. Future meta-analysis with additional study datasets will be helpful in prioritizing the reliable findings that can be generalized across studies.

\section{Methods \\ Alzheimer's disease neuroimaging initiative}

Data analyzed in this study were obtained from the ADNI database [68]. The ADNI was launched in 2003 by the National Institute on Aging, the National Institute of Biomedical Imaging and Bioengineering, the United States Food and Drug Administration, private pharmaceutical companies, and nonprofit organizations as a $\$ 60$ million, 5 -year public-private partnership. The primary goal of the ADNI study has been to test whether serial magnetic resonance imaging, positron emission tomography, other biological markers, and clinical and neuropsychological assessments can be combined to measure the progression of MCI and early AD. The data from ADNIMERGE R package, dated September 14, 2018, together with epigenetic data and REGISTRY.csv file (http://adni.loni. usc.edu) were used for the analyses reported here. The ADNIMERGE table merges several key variables from various case report forms and biomarker lab summaries across the ADNI protocols (ADNI1, ADNIGO, and ADNI2).

\section{DNA methylation profile}

DNA methylation profiles were generated at AbbVie, Inc. from blood samples of ADNI participants using Infinium $^{\circledR}$ MethylationEPIC BeadChip (Illumina, Inc., San Diego, CA, USA). Genomic DNA samples obtained from National Cell Repository for Alzheimer's Disease were bisulfite-converted using the EZ-DNA Methylation Kits (Zymo Research, Irvine, CA, USA) and subsequently analyzed using the Illumina Infinium ${ }^{\circledR} \mathrm{HD}$ methylation protocol on the HiScan ${ }^{\mathrm{TM}}$ system (Illumina, Inc). Detailed sample-level quality control was described previously [20]. A probe-level quality control was performed as well; probes that did not perform well (probes with detection 
$P$ value $\geq 0.05$ in $\geq 1 \%$ samples $[n=3047]$ and probes with bead count $<3$ in $\geq 5 \%$ of samples $[n=1011]$ ) were filtered out. A total of 863,718 probes were used in the downstream analysis. Methylation profiles from 653 baseline DNA samples were used in this study. Participants with reverse conversion (e.g., from $\mathrm{CN}$ to $\mathrm{MCI}$ and back to $\mathrm{CN}$ ) or conversion from $\mathrm{CN}$ to $\mathrm{AD}$, those with a diagnosis of $\mathrm{AD}$ at baseline, and those without post-baseline measurements were excluded, leaving a total of 519 samples reported in the study.

\section{Disease progression phenotype definition}

The DNA samples were not necessarily collected at the same time point as the baseline visit for the main ADNI clinical study. Considering that the ADNI epigenetic dataset is a longitudinal analysis of DNA samples, up to 5 samples per participant were available, and the sample from the earliest time point was considered the baseline data. Participants were required to have a follow-up clinical assessment from at least one post-baseline visit.

Disease progression from the baseline of this study was defined using two continuous endpoints for $\mathrm{CN}$ participants and one continuous endpoint for patients with MCI; both groups also had a dichotomized endpoint (presence or absence of conversion). The original PACC, which was designed to capture 3 key domains of episodic memory, executive function, and orientation, is a composite of 4 component scores: total recall score, delayed recall score, Digit Symbol Substitution Test (DSST) score, and MMSE total score [26]. The ADNIMERGE database includes a modified PACC (mPACC trailsB; a standardized $Z$-score composite of MMSE, logical memory delayed recall, Alzheimer Disease Assessment Scale-Cognitive Subscale delayed word recall, and Trail-Making Test B [27, 69]) and an additional variant of the modified PACC that uses the original component score for DSST (mPACC $\mathrm{migit}_{\text {iit }}$ [70]; both $\mathrm{mPACC}_{\text {trailsB }}$ and $\mathrm{mPACC}_{\text {digit }}$ were used in this study. For the continuous endpoints, the rate of cognitive decline was measured by the slopes of mPACC $_{\text {digit }}[26,71]$ and MPACC $_{\text {trailsB }}[72]$ in $\mathrm{CN}$ participants and by the slope of CDR-SB in patients with MCI [73]. For the dichotomized endpoint, a converter status was assigned if there was a conversion of clinical diagnosis (from $\mathrm{CN}$ to $\mathrm{MCI}$ or from $\mathrm{MCI}$ to $\mathrm{AD}$ ) at a post-baseline visit. If the diagnosis was undefined at the DNA sampling visit, but the clinical diagnosis had been consistent before and after the DNA sampling visit, the diagnosis at DNA sampling was assumed to be the same as that at the adjacent visits.

\section{Identification of DMPs}

M-value, which provides higher detection rates and true positive rates for both highly methylated and unmethylated $\mathrm{CpG}$ sites and is considered statistically more valid than beta-value [74], was used to identify DMPs using R package limma [75]. The statistical model, adjusted for age at DNA sampling, sex, cell composition, DNA source (buffy coat vs. whole blood), was used to analyze rate of cognitive decline or conversion status. Since the study was prone to significant inflation and bias of test statistics, we applied a Bayesian method in R package BACON v1.10.1 to control for inflation of test statistics and for lambda inflation factors before and after correction were reported; the Bayesian method is based on estimation of the empirical null distribution [31]. A stringent threshold using Bonferroni correction was used to declare study-wide significance. The discovered DMPs for each endpoint were assessed for consistency in 2 ways: (1) the effect size and directionality were compared between the continuous and dichotomized endpoints, and (2) the effect sizes from this study were compared with those reported in a recent meta-analysis in brain [35] and several EWAS studies in blood [20, 34], recognizing that the phenotype in the two studies was different.

\section{Identification and annotation of DMRs and genomic feature enrichment}

DMRs were identified using comb-p [76] with a distance of $500 \mathrm{bp}$ and a seeded $P$ value of $1.0 \times 10^{-4}$. The DMR analyses were carried out for all probes, irrespective of the directionality of the differential methylation, and DMRs with at least 3 probes and Sidak corrected $P<0.05$ were considered significant and are reported. Comb-p identifies regions enriched for probes with low $P$ values using the Stouffer-Liptak method to correct for autocorrelation and adjusts for multiple testing using the Sidak correction. The identified DMRs were annotated by HOMER software [77]. HOMER first determines the distance of a DMR to the nearest transcription start site and assigns the DMR to that gene, and it determines the genomic annotation of the region occupied by the center of the DMR.

To provide additional evidence for the DMR analysis, a secondary DMR calling algorithm DMRcate was employed [78]. The algorithm used the default parameters, except for the following: the robust regression for limma was used to be consistent with the DMP analysis, the test statistics after BACON statistics were used with a pcutoff at $1.0 \times 10^{-4}$, and a minimum of $3 \mathrm{CpG}$ probes were applied for DMR calling. DMRcate calculates two smoothed estimates (one based on the square of moderated limma t statistic and the other null model) using a Satterthwaite approximation to compare these estimates and adjusts for multiple testing using the FDR method. Both sets of DMR results are reported and compared for 
consistency, but the comb-p analysis was considered as the primary analysis.

The identified DMRs were also compared to 2 lists of DMRs reported previously to be associated with conversion to $\mathrm{AD}[25,33]$.

\section{Gene set enrichment and overrepresentation analyses}

Using R package, methylGSA, GSEA analysis was conducted that used Robust Rank Aggregation to adjust for multiple $P$ values of each gene and applied pre-ranked version of GSEA in gene set testing $[79,80]$. The following gene ontology databases were used: subsets of Molecular Signatures Database, KEGG database, and c2.cp v7.0 (includes a superset of c2.cp.biocarta, c2.cp.kegg, and c2.cp.reactome among others) [81-83]. Over-representation analysis was performed using R package missMethyl [84] to test for pathway enrichment using a hypergeometric test, taking into account the number of $\mathrm{CpG}$ sites per gene on the EPIC array.

\section{Co-occurrence of biological concepts and genes using Euretos Al Platform}

Since the database gene assignment to ontology terms could be incomplete, we also applied co-occurrence analysis of genes and key biological concepts, such as neurogenesis, amyloid, tau proteins, and cognition, to identify key genes annotated by DMRs (using the comb-p approach) with prior evidence associated with these terms using Euretos AI Platform (Netherlands). Synonyms were also used in the search; therefore, such analysis could result in false positive findings. With the relationship pairs with 5 or more references, we followed up with a human curation of abstracts, and false positives were flagged!

\begin{abstract}
Abbreviations
AD: Alzheimer's disease; ADNI: Alzheimer's Disease Neuroimaging Initiative; CDR-SB: Clinical Dementia Rating Scale Sum of Boxes; CN: Cognitively normal; CpG: Cytosine-phosphate-guanine; CSF: Cerebrospinal fluid; DMP: Differentially methylated position; DMR: Differentially methylated region; EWAS: Epigenome-wide association study; $\mathrm{MCl}$ : Mild cognitive impairment; MMSE: Mini-Mental State Examination; mPACC: Modified Preclinical Alzheimer Cognitive Composite; mPACC $_{\text {digit }}$ MPACC that used Digit Symbol Substitution Test; mPACC $_{\text {trailsB }}$ : MPACC that used Trail-Making Test Part B; mQTL: Methylation quantitative trait locus; NCRAD: National Centralized Repository for Alzheimer's Disease and Related Dementias; SNP: Single nucleotide polymorphism.
\end{abstract}

\section{Supplementary Information}

The online version contains supplementary material available at https://doi. org/10.1186/s13148-021-01179-2.

Additional file 1: Supplemental Figures.

Additional file 2: Supplemental Tables.

\section{Acknowledgements}

Samples from the NCRAD, which receives government support under a cooperative agreement Grant (U24 AG21886) awarded by the National Institute on Aging, were used in this study. We thank contributors who collected samples used in this study, as well as study participants and their families, whose help and participation made this work possible. Authors thank Kelley Faber, Kelly Nudelman, and Tatiana M. Foroud of NCRAD for performing the DNA plating, QC, and shipping service to enable the epigenetic data generation. Editorial assistance was provided by Narender Dhingra, MBBS, PhD, CMPP, of System One, and funded by Janssen Global Services, LLC.

\section{Authors' contributions}

The investigators within the ADNI contributed to the design and implementation of ADNl and/or provided data but did not participate in analysis or writing of this report. A complete listing of ADNI investigators can be found at: http:// adni.loni.usc.edu/wp-content/uploads/how_to_apply/ADNI_Acknowledg ement_List.pdf. AV, JWD, KI, JFW, AJS, KN, and QSL contributed to the study concept and design. KI contributed to data acquisition, and QSL analyzed data. All authors read, provided feedback, and approved the final manuscript.

\section{Funding}

Data collection and sharing for this project was funded by the Alzheimer's Disease Neuroimaging Initiative (ADNI) (National Institutes of Health Grant U01 AG024904) and DOD ADNI (Department of Defense Award Number W81XWH-12-2-0012). ADNI is funded by the National Institute on Aging, the National Institute of Biomedical Imaging and Bioengineering, and through generous contributions from the following: AbbVie, Alzheimer's Association; Alzheimer's Drug Discovery Foundation; Araclon Biotech; BioClinica, Inc:; Biogen; Bristol-Myers Squibb Company; CereSpir, Inc.; Cogstate; Eisai Inc.; Elan Pharmaceuticals, Inc.; Eli Lilly and Company; Eurolmmun; F. Hoffmann-La Roche Ltd and its affiliated company Genentech, Inc:; Fujirebio; GE Healthcare; IXICO Ltd.; Janssen Alzheimer Immunotherapy Research \& Development, LLC.; Johnson \& Johnson Pharmaceutical Research \& Development LLC.; Lumosity; Lundbeck; Merck \& Co., Inc.; Meso Scale Diagnostics, LLC.; NeuroRx Research; Neurotrack Technologies; Novartis Pharmaceuticals Corporation; Pfizer Inc.; Piramal Imaging; Servier; Takeda Pharmaceutical Company; and Transition Therapeutics. The Canadian Institutes of Health Research is providing funds to support ADNI clinical sites in Canada. Private sector contributions are facilitated by the Foundation for the National Institutes of Health (www.fnih. org). The grantee organization is the Northern California Institute for Research and Education, and the study is coordinated by the Alzheimer's Therapeutic Research Institute at the University of Southern California. ADNI data are disseminated by the Laboratory for Neuro Imaging at the University of Southern California. The epigenetic data generation was made possible by contributions of laboratory service, EPIC arrays, reagents, and funding by AbbVie, Janssen Research \& Development, LLC, and Biogen. DNA banking and preparation was in part supported by the National Centralized Repository for Alzheimer's Disease (NCRAD; U24 AG21886). Additional support was provided by NIA grants P30 AG010133, P30 AG072976, R01 AG019771, R01 AG068193, R03 AG054936, and U01 AG068057, and NLM grants R01 LM012535 and R01 LM013463.

\section{Availability of data and materials}

Data used in preparation of this article were obtained from the ADNI database (adni.loni.usc.edu). ADNI data are disseminated by the Laboratory for Neuro Imaging at the University of Southern California.

\section{Declarations}

\section{Ethics approval and consent to participate}

The institutional review boards of all participating ADNI sites reviewed and approved the data collection protocol provided by ADNI. For up-to-date information, see www.adni-info.org.

\section{Consent for publication}

Not applicable.

\section{Competing interests}

QSL is an employee of Janssen Research \& Development, LLC, and may own stock and/or stock options in Johnson \& Johnson. AV, JWD, KI and JFW are employees of AbbVie and may own stock and/or stock options. KN reports 
no competing interests. AJS receives support from multiple $\mathrm{NIH}$ grants. He has also received support from Avid Radiopharmaceuticals, a subsidiary of El Lilly (in kind contribution of PET tracer precursor), Bayer Oncology (Scientific Advisory Board), and Springer-Nature Publishing (Editorial Office Support as Editor-in-Chief of Brain Imaging and Behavior.

\section{Author details}

${ }^{1}$ Neuroscience, Janssen Research and Development, LLC, 1125 Trenton-Harbourton Road, Titusville, NJ 08560, USA. ${ }^{2}$ Genomics Research Center, AbbVie, North Chicago, IL, USA. Indiana Alzheimer's Disease Research Center, Department of Radiology and Imaging Sciences, Indiana University School of Medicine, Indianapolis, IN, USA.

Received: 5 April 2021 Accepted: 29 September 2021

Published online: 15 October 2021

\section{References}

1. Cummings JL, Morstorf T, Zhong K. Alzheimer's disease drug-development pipeline: few candidates, frequent failures. Alzheimers Res Ther. 2014;6:37.

2. Citeline: Informa Pharma Intelligence. https://citeline.informa.com/trials/ results?ald=e0f54816-1 cac-4434-a6ea-296c934bc8fc. Accessed June 9.

3. Alzheimer's Association. FDA-approved treatments for Alzheimer's. https://www.alz.org/media/documents/fda-approved-treatments-alzhe imers-ts.pdf. Accessed June 9.

4. Kabir MT, Uddin MS, Mamun AA, Jeandet P, Aleya L, Mansouri RA, et al. Combination drug therapy for the management of Alzheimer's disease. Int J Mol Sci. 2020;21.

5. Yiannopoulou KG, Papageorgiou SG. Current and future treatments in Alzheimer disease: an update. J Cent Nerv Syst Dis. 2020;12:1179573520907397.

6. Cummings J, Lee G, Zhong K, Fonseca J, Taghva K. Alzheimer's disease drug development pipeline: 2021. Alzheimers Dement. 2021;7:e12179.

7. U.S. Food and Drug Administration. FDA grants accelerated approval for Alzheimer's drug. https://www.fda.gov/news-events/press-announceme nts/fda-grants-accelerated-approval-alzheimers-drug. Accessed June 9.

8. Veitch DP, Weiner MW, Aisen PS, Beckett LA, Cairns NJ, Green RC, et al. Understanding disease progression and improving Alzheimer's disease clinical trials: recent highlights from the Alzheimer's Disease Neuroimaging Initiative. Alzheimers Dement. 2019;15:106-52.

9. Llano DA, Bundela S, Mudar RA, Devanarayan V, Alzheimer's Disease Neuroimaging I. A multivariate predictive modeling approach reveals a novel CSF peptide signature for both Alzheimer's Disease state classification and for predicting future disease progression. PLOS ONE. 2017;12:e0182098.

10. Ortner M, Drost R, Heddderich D, Goldhardt O, Muller-Sarnowski F, DiehlSchmid J, et al. Amyloid PET, FDG-PET or MRI? The power of different imaging biomarkers to detect progression of early Alzheimer's disease. BMC Neurol. 2019;19:264.

11. Yagi T, Kanekiyo M, Ito J, Ihara R, Suzuki K, Iwata A, et al. Identification of prognostic factors to predict cognitive decline of patients with early Alzheimer's disease in the Japanese Alzheimer's Disease Neuroimaging Initiative study. Alzheimers Dement (N Y). 2019;5:364-73.

12. Webers A, Heneka MT, Gleeson PA. The role of innate immune responses and neuroinflammation in amyloid accumulation and progression of Alzheimer's disease. Immunol Cell Biol. 2020;98:28-41.

13. Pillai JA, Bena J, Bebek G, Bekris LM, Bonner-Jackson A, Kou L, et al. Inflammatory pathway analytes predicting rapid cognitive decline in $\mathrm{MCl}$ stage of Alzheimer's disease. Ann Clin Transl Neurol. 2020;7:1225-39.

14. Albright J. Forecasting the progression of Alzheimer's disease using neural networks and a novel preprocessing algorithm. Alzheimers Dement $(\mathrm{N}$ Y). 2019;5:483-91.

15. Beltran JF, Wahba BM, Hose N, Shasha D, Kline RP, Alzheimer's Disease Neuroimaging I. Inexpensive, non-invasive biomarkers predict Alzheimer transition using machine learning analysis of the Alzheimer's Disease Neuroimaging (ADNI) database. PLoS ONE. 2020;15:e0235663.

16. Moradi E, Pepe A, Gaser C, Huttunen H, Tohka J, Alzheimer's Disease Neuroimaging I. Machine learning framework for early MRI-based Alzheimer's conversion prediction in MCl subjects. Neuroimage. 2015;104:398-412.
17. Meder B, Haas J, Sedaghat-Hamedani F, Kayvanpour E, Frese K, Lai A, et al. Epigenome-wide association study identifies cardiac gene patterning and a novel class of biomarkers for heart failure. Circulation. 2017;136:1528-44.

18. Pan Y, Liu G, Zhou F, Su B, Li Y. DNA methylation profiles in cancer diagnosis and therapeutics. Clin Exp Med. 2018;18:1-14.

19. Somineni HK, Venkateswaran S, Kilaru V, Marigorta UM, Mo A, Okou DT, et al. Blood-derived DNA methylation signatures of Crohn's disease and severity of intestinal inflammation. Gastroenterology. 2019;156:2254-65.

20. Vasanthakumar A, Davis JW, Idler K, Waring JF, Asque E, Riley-Gillis B, et al. Harnessing peripheral DNA methylation differences in the Alzheimer's Disease Neuroimaging Initiative (ADNI) to reveal novel biomarkers of disease. Clin Epigenet. 2020;12:84.

21. Madrid A, Hogan KJ, Papale LA, Clark LR, Asthana S, Johnson SC, et al. DNA hypomethylation in blood links B3GALT4 and ZADH2 to Alzheimer's disease. J Alzheimers Dis. 2018;66:927-34.

22. Kobayashi N, Shinagawa S, Nagata T, Shimada K, Shibata N, Ohnuma T, et al. Development of biomarkers based on DNA methylation in the NCAPH2/LMF2 promoter region for diagnosis of Alzheimer's disease and amnesic mild cognitive impairment. PLoS ONE. 2016;11:e0146449.

23. Lunnon K, Smith R, Hannon E, De Jager PL, Srivastava G, Volta M, et al. Methylomic profiling implicates cortical deregulation of ANK1 in Alzheimer's disease. Nat Neurosci. 2014;17:1164-70.

24. Chouliaras L, Pishva E, Haapakoski R, Zsoldos E, Mahmood A, Filippini $\mathrm{N}$, et al. Peripheral DNA methylation, cognitive decline and brain aging: pilot findings from the Whitehall II imaging study. Epigenomics. 2018;10:585-95.

25. Lardenoije R, Roubroeks JAY, Pishva E, Leber M, Wagner $H$, latrou A, et al. Alzheimer's disease-associated (hydroxy)methylomic changes in the brain and blood. Clin Epigenet. 2019;11:164.

26. Donohue MC, Sperling RA, Salmon DP, Rentz DM, Raman R, Thomas RG, et al. The preclinical Alzheimer cognitive composite: measuring amyloidrelated decline. JAMA Neurol. 2014;71:961-70.

27. Donohue MC, Sperling RA, Petersen R, Sun CK, Weiner MW, Aisen PS, et al. Association between elevated brain amyloid and subsequent cognitive decline among cognitively normal persons. JAMA. 2017;317:2305-16.

28. Lynch CA, Walsh C, Blanco A, Moran M, Coen RF, Walsh JB, et al. The clinical dementia rating sum of box score in mild dementia. Dement Geriatr Cogn Disord. 2006;21:40-3.

29. Cedarbaum JM, Jaros M, Hernandez C, Coley N, Andrieu S, Grundman M, et al. Rationale for use of the clinical dementia rating sum of boxes as a primary outcome measure for Alzheimer's disease clinical trials. Alzheimers Dement. 2013;9:S45-55.

30. Williams MM, Storandt M, Roe CM, Morris JC. Progression of Alzheimer's disease as measured by Clinical Dementia Rating Sum of Boxes scores. Alzheimers Dement. 2013;9:S39-44.

31. van Iterson M, van Zwet EW, Consortium B, Heijmans BT. Controlling bias and inflation in epigenome- and transcriptome-wide association studies using the empirical null distribution. Genome Biol. 2017;18:19.

32. Volkov P, Olsson AH, Gillberg L, Jorgensen SW, Brons C, Eriksson KF, et al. A genome-wide $\mathrm{mQTL}$ analysis in human adipose tissue identifies genetic variants associated with DNA methylation, gene expression and metabolic traits. PLoS ONE. 2016;11:e0157776.

33. Roubroeks JAY, Smith AR, Smith RG, Pishva E, Ibrahim Z, Sattlecker M, et al. An epigenome-wide association study of Alzheimer's disease blood highlights robust DNA hypermethylation in the HOXB6 gene. Neurobiol Aging. 2020;95:26-45.

34. Starnawska A, Tan Q, McGue M, Mors O, Borglum AD, Christensen K, et al. Epigenome-wide association study of cognitive functioning in middleaged monozygotic twins. Front Aging Neurosci. 2017;9:413.

35. Smith RG, Pishva E, Shireby G, Smith AR, Roubroeks JAY, Hannon E, et al. A meta-analysis of epigenome-wide association studies in Alzheimer's disease highlights novel differentially methylated loci across cortex. Nat Commun. 2021;12:3517.

36. Gavalas A, Ruhrberg C, Livet J, Henderson CE, Krumlauf R. Neuronal defects in the hindbrain of Hoxa1, Hoxb1 and Hoxb2 mutants reflect regulatory interactions among these Hox genes. Development. 2003;130:5663-79.

37. Gaufo GO, Thomas KR, Capecchi MR. Hox3 genes coordinate mechanisms of genetic suppression and activation in the 
generation of branchial and somatic motoneurons. Development. 2003;130:5191-201.

38. Davenne M, Maconochie MK, Neun R, Pattyn A, Chambon P, Krumlauf $\mathrm{R}$, et al. Hoxa2 and Hoxb2 control dorsoventral patterns of neuronal development in the rostral hindbrain. Neuron. 1999;22:677-91.

39. Lowe LA, Yamada S, Kuehn MR. HoxB6-Cre transgenic mice express Cre recombinase in extra-embryonic mesoderm, in lateral plate and limb mesoderm and at the midbrain/hindbrain junction. Genesis. 2000;26:118-20.

40. De Jager PL, Srivastava G, Lunnon K, Burgess J, Schalkwyk LC, Yu L, et al. Alzheimer's disease: early alterations in brain DNA methylation at ANK1, BIN1, RHBDF2 and other loci. Nat Neurosci. 2014;17:1156-63.

41. Li QS, Sun Y, Wang T. Epigenome-wide association study of Alzheimer's disease replicates 22 differentially methylated positions and 30 differentially methylated regions. Clin Epigenet. 2020;12:149.

42. Smith RG, Hannon E, De Jager PL, Chibnik L, Lott SJ, Condliffe D, et al. Elevated DNA methylation across a 48-kb region spanning the HOXA gene cluster is associated with Alzheimer's disease neuropathology. Alzheimers Dement. 2018;14:1580-8.

43. Gasparoni G, Bultmann S, Lutsik P, Kraus TFJ, Sordon S, Vlcek J, et al. DNA methylation analysis on purified neurons and glia dissects age and Alzheimer's disease-specific changes in the human cortex. Epigenet Chromatin. 2018;11:41.

44. Labadorf A, Hoss AG, Lagomarsino V, Latourelle JC, Hadzi TC, Bregu $J$, et al. RNA sequence analysis of human Huntington disease brain reveals an extensive increase in inflammatory and developmental gene expression. PLoS ONE. 2015;10:e0143563.

45. Evangelisti C, Bianco F, Pradella LM, Puliti A, Goldoni A, Sbrana I, et al. Apolipoprotein B is a new target of the GDNF/RET and ET-3/EDNRB signalling pathways. Neurogastroenterol Motil. 2012;24:e497-508.

46. Sanchez-Mut JV, Glauser L, Monk D, Graff J. Comprehensive analysis of PM20D1 QTL in Alzheimer's disease. Clin Epigenet. 2020;12:20.

47. Sanchez-Mut JV, Heyn H, Silva BA, Dixsaut L, Garcia-Esparcia P, Vidal E, et al. PM20D1 is a quantitative trait locus associated with Alzheimer's disease. Nat Med. 2018;24:598-603.

48. Sanchez-Mut JV, Aso E, Heyn H, Matsuda T, Bock C, Ferrer I, et al. Promoter hypermethylation of the phosphatase DUSP22 mediates PKAdependent TAU phosphorylation and CREB activation in Alzheimer's disease. Hippocampus. 2014;24:363-8.

49. Luna-Munoz J, Chavez-Macias L, Garcia-Sierra F, Mena R. Earliest stages of tau conformational changes are related to the appearance of a sequence of specific phospho-dependent tau epitopes in Alzheimer's disease. J Alzheimers Dis. 2007;12:365-75.

50. Lonze BE, Riccio A, Cohen S, Ginty DD. Apoptosis, axonal growth defects, and degeneration of peripheral neurons in mice lacking CREB. Neuron. 2002;34:371-85.

51. Querfurth HW, LaFerla FM. Alzheimer's disease. N Engl J Med. 2010;362:329-44

52. Gene Set Enrichment Analysis. Gene set: CEBPGAMMA_Q6. https:// www.gsea-msigdb.org/gsea/msigdb/cards/CEBPGAMMA_Q6. Accessed June 17.

53. Tian X, Qin Y, Tian Y, Ge X, Cui J, Han H, et al. Identification of vascular dementia and Alzheimer's disease hub genes expressed in the frontal lobe and temporal cortex by weighted co-expression network analysis and construction of a protein-protein interaction. Int J Neurosci. 2021:1-12.

54. Choi JY, Cho SJ, Park JH, Yun SM, Jo C, Kim EJ, et al. Elevated cerebrospinal fluid and plasma N-Cadherin in Alzheimer disease. J Neuropathol Exp Neurol. 2020;79:484-92.

55. Srinivasan K, Friedman BA, Etxeberria A, Huntley MA, van der Brug MP, Foreman $\mathrm{O}$, et al. Alzheimer's patient microglia exhibit enhanced aging and unique transcriptional activation. Cell Rep. 2020;31:107843.

56. Bonham LW, Sirkis DW, Yokoyama JS. The transcriptional landscape of microglial genes in aging and neurodegenerative disease. Front Immunol. 2019;10:1170.

57. Bellenguez C, Küçükali F, Jansen I, Andrade V, Moreno-Grau S, Amin N, et al. New insights on the genetic etiology of Alzheimer's and related dementia. medRxiv. 2020:2020.10.01.20200659.

58. Wightman DP, Jansen IE, Savage JE, Shadrin AA, Bahrami S, Rongve A, et al. Largest GWAS $(N=1,126,563)$ of Alzheimer's disease implicates microglia and immune cells. medRxiv. 2020:2020.11.20.20235275.
59. Kim Y, Park J, Choi YK. The role of astrocytes in the central nervous system focused on BK channel and heme oxygenase metabolites: a review. Antioxidants (Basel). 2019:8:121.

60. Heaney CF, Kinney JW. Role of GABA(B) receptors in learning and memory and neurological disorders. Neurosci Biobehav Rev. 2016;63:1-28.

61. Mudo G, Bonomo A, Di Liberto V, Frinchi M, Fuxe K, Belluardo N. The FGF-2/FGFRs neurotrophic system promotes neurogenesis in the adult brain. J Neural Transm (Vienna). 2009;1 16:995-1005.

62. Chen W, Wu L, Hu Y, Jiang L, Liang N, Chen J, et al. MicroRNA-107 ameliorates damage in a cell model of Alzheimer's disease by mediating the FGF7/FGFR2/PI3K/Akt pathway. J Mol Neurosci. 2020.

63. El Hajj N, Dittrich M, Bock J, Kraus TF, Nanda I, Muller T, et al. Epigenetic dysregulation in the developing Down syndrome cortex. Epigenetics. 2016;11:563-78.

64. Young Jl, Sivasankaran SK, Wang L, Ali A, Mehta A, Davis DA, et al. Genome-wide brain DNA methylation analysis suggests epigenetic reprogramming in Parkinson disease. Neurol Genet. 2019;5:e342.

65. Bouter Y, Kacprowski T, Weissmann R, Dietrich K, Borgers H, Brauss A, et al. Deciphering the molecular profile of plaques, memory decline and neuron loss in two mouse models for Alzheimer's disease by deep sequencing. Front Aging Neurosci. 2014;6:75.

66. Bencze J, Morotz GM, Seo W, Bencs V, Kalman J, Miller CCJ, et al. Biological function of Lemur tyrosine kinase 2 (LMTK2): implications in neurodegeneration. Mol Brain. 2018;11:20.

67. Jack CR Jr, Knopman DS, Jagust WJ, Petersen RC, Weiner MW, Aisen PS, et al. Tracking pathophysiological processes in Alzheimer's disease: an updated hypothetical model of dynamic biomarkers. Lancet Neurol. 2013;12:207-16.

68. Cucinotta D, Vanelli M. WHO declares COVID-19 a pandemic. Acta Biomed. 2020;91:157-60.

69. Reitan RM, Wolfson D. The Halstead-Reitan neuropsychological test battery: theory and clinical interpretation. 2nd ed. Tucson: Neuropsychology Press; 1993.

70. Kiely KM, Butterworth P, Watson N, Wooden M. The Symbol Digit Modalities Test: normative data from a large nationally representative sample of Australians. Arch Clin Neuropsychol. 2014;29:767-75.

71. Jaeger J. Digit symbol substitution test: the case for sensitivity over specificity in neuropsychological testing. J Clin Psychopharmacol. 2018;38:513-9.

72. Salthouse TA. What cognitive abilities are involved in trail-making performance? Intelligence. 2011;39:222-32.

73. Snider BJ, Fagan AM, Roe C, Shah AR, Grant EA, Xiong C, et al. Cerebrospinal fluid biomarkers and rate of cognitive decline in very mild dementia of the Alzheimer type. Arch Neurol. 2009;66:638-45.

74. Du P, Zhang X, Huang CC, Jafari N, Kibbe WA, Hou L, et al. Comparison of Beta-value and $\mathrm{M}$-value methods for quantifying methylation levels by microarray analysis. BMC Bioinform. 2010;11:587.

75. Ritchie ME, Phipson B, Wu D, Hu Y, Law CW, Shi W, et al. limma powers differential expression analyses for RNA-sequencing and microarray studies. Nucleic Acids Res. 2015;43:e47.

76. Pedersen BS, Schwartz DA, Yang IV, Kechris KJ. Comb-p: software for combining, analyzing, grouping and correcting spatially correlated P-values. Bioinformatics. 2012;28:2986-8.

77. Heinz S, Benner C, Spann N, Bertolino E, Lin YC, Laslo P, et al. Simple combinations of lineage-determining transcription factors prime cisregulatory elements required for macrophage and $\mathrm{B}$ cell identities. Mol Cell. 2010;38:576-89.

78. Peters TJ, Buckley MJ, Statham AL, Pidsley R, Samaras K, Lord RV, et al. De novo identification of differentially methylated regions in the human genome. Epigenet Chromatin. 2015;8:6.

79. Ren X, Kuan PF. methylGSA: a Bioconductor package and Shiny app for DNA methylation data length bias adjustment in gene set testing. Bioinformatics. 2019;35:1958-9.

80. Subramanian A, Tamayo P, Mootha VK, Mukherjee S, Ebert BL, Gillette MA, et al. Gene set enrichment analysis: a knowledge-based approach for interpreting genome-wide expression profiles. Proc Natl Acad Sci USA. 2005;102:15545-50

81. Fabregat A, Jupe S, Matthews L, Sidiropoulos K, Gillespie M, Garapati $P$, et al. The reactome pathway knowledgebase. Nucleic Acids Res. 2018:46:D649-55. 
82. Kanehisa M, Furumichi M, Tanabe M, Sato Y, Morishima K. KEGG: new perspectives on genomes, pathways, diseases and drugs. Nucleic Acids Res. 2017;45:D353-61.

83. Liberzon A, Subramanian A, Pinchback R, Thorvaldsdottir H, Tamayo P, Mesirov JP. Molecular signatures database (MSigDB) 3.0. Bioinformatics. 2011;27:1739-40.

84. Phipson B, Maksimovic J, Oshlack A. missMethyl: an R package for analyzing data from Illumina's HumanMethylation450 platform. Bioinformatics. 2016;32:286-8.

\section{Publisher's Note}

Springer Nature remains neutral with regard to jurisdictional claims in published maps and institutional affiliations.
Ready to submit your research? Choose BMC and benefit from:

- fast, convenient online submission

- thorough peer review by experienced researchers in your field

- rapid publication on acceptance

- support for research data, including large and complex data types

- gold Open Access which fosters wider collaboration and increased citations

- maximum visibility for your research: over $100 \mathrm{M}$ website views per year

At BMC, research is always in progress.

Learn more biomedcentral.com/submissions 